Measur ement and vi sual i zat $i$ on of three- di mensi onal $r$ adi al and vect or ed magnet i $c$ $\mathrm{fi}$ el d di stribution by use of the magnet i $\mathrm{c}$ CT met hod

\begin{tabular}{|l|l|}
\hline 著者 & $\begin{array}{l}\text { N shi mur a T., M yantt o Y., Yanada Sot oshi , } \\
\text { I wahar a Masayoshi }\end{array}$ \\
\hline $\begin{array}{l}\text { j our nal or } \\
\text { publ i cat i on ti t l e }\end{array}$ & I EEE Tr ansact i ons on Nagnet i cs \\
\hline vol une & 41 \\
\hline number & 10 \\
\hline page r ange & $3739-3741$ \\
\hline year & 2005- 10-01 \\
\hline URL & ht t p: //hdl . handl e. net /2297/6906 \\
\hline
\end{tabular}




\title{
Measurement and Visualization of Three-Dimensional Radial and Vectored Magnetic Field Distribution by Use of the Magnetic CT Method
}

\author{
T. Nishimura, Y. Miyamoto, S. Yamada, Member, IEEE, and M. Iwahara, Member, IEEE
}

Graduate School of Natural Science and Technology, Institute of Nature and Environmental Technology, Kanazawa University, Ishikawa 920-1192, Japan

\begin{abstract}
The measurement and visualization of three-dimensional radial and vectored magnetic field distribution based on the magnetic computed tomography (CT) method have been reported. Applying the method, it is possible to measure a surrounding magnetic field such as a spherical region by using a simple probe, which is not limited to the measurement of two-dimensional magnetic field distribution. It has been shown that the magnetic field distribution of a spherical surface covering a sample object can be visualized from the measured and calculated results with a spherical CT probe and CT reconstruction algorithm.
\end{abstract}

Index Terms-Magnetic computed tomography (CT) method, magnetic field measurement, spherical magnetic CT probe, spherical magnetic field visualization.

\section{INTRODUCTION}

$\mathbf{T}$ HERE ARE many kinds of magnetic sensors, i.e., Fluxgate sensors, MI and MR sensors, and superconducting quantum interference device (SQUID) sensors for measuring a magnetic field. These are capable of also measuring dc fields. In order to know a surrounding magnetic field of an object, usually a scanning measurement is performed repetitively with these sensors. However, this method has a problem in the case of measuring narrow areas of equipment inside an air gap due to the size of measurement equipment. Moreover, a scanning measurement needs a lot of measuring time. To solve this problem, the computed tomography (CT) method [1], [2] is applicable. By use of this method, with a simple probe that is called the magnetic CT probe [3], an ac magnetic field is easily measured and visualized at a time. The principle of two-dimensional (2-D) magnetic CT algorithms has been reported so far. Furthermore, as a development of a multidimensional magnetic driving system such as a spherical surface motor [4], the measurement with no limitation to the plane distribution will be requested in future. In this paper, we propose the new idea of measurement magnetic flux distribution on a curved surface, as shown in Fig. 1. Then, the advanced principles of measurement radial and vectored magnetic fields are shown, and a simple experiment is carried out to measure and visualize magnetic field distribution from the magnetic source with a spherical magnetic CT probe.

\section{RECONSTRUCTION ALGORITHM}

As is well known, the projection of an unknown field $f(x, y)$ is obtained by the following parallel line integral equation, where the field can be easily reconstructed and

Digital Object Identifier 10.1109/TMAG.2005.854259

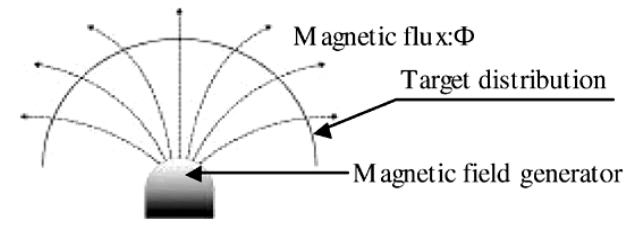

Fig. 1. Measuring target of magnetic flux distribution.

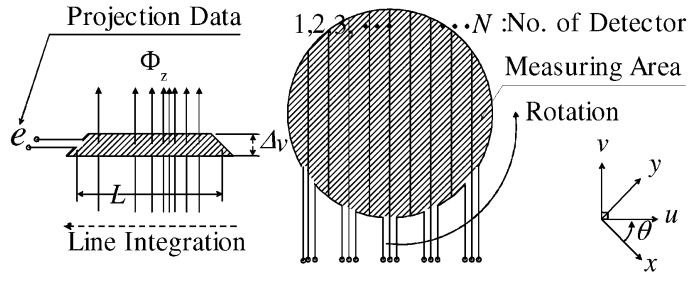

Fig. 2. Two-dimensional magnetic $\mathrm{CT}$ probe [3] and its principle.

visualized after a proper signal processing is utilized to this projection $p$

$$
\begin{aligned}
p & \equiv \int_{u} f(x, y) d u \\
\left(\begin{array}{l}
u \\
v
\end{array}\right) & =\left(\begin{array}{ll}
\cos \theta & \sin \theta \\
-\sin \theta & \cos \theta
\end{array}\right)\left(\begin{array}{l}
x \\
y
\end{array}\right)
\end{aligned}
$$

where $p$ is the projection of the unknown field along the line $u$, and $u, v$ are the rotated coordinates of $x$ and $y$ with $\theta$ as the angle in Fig. 2.

In the reconstruction processing from obtained projection data, the filtered back projection (FBP) algorithm [2] is utilized generally, which is one of the reconstruction techniques.

Accordingly, after the whole projection is scanned around the field, obtained projection data is resolved numerically by use of the FBP method.

Applying this method to the magnetic field, the line integration of magnetic flux distribution $B(x, y)$ can be obtained as an induced ac voltage between terminals of every each slender coil shown in Fig. 2. This figure also introduces the magnetic CT 


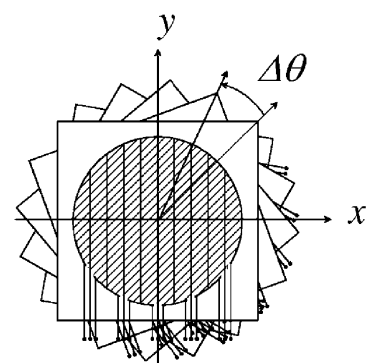

Fig. 3. Multilayered magnetic CT probe [5].

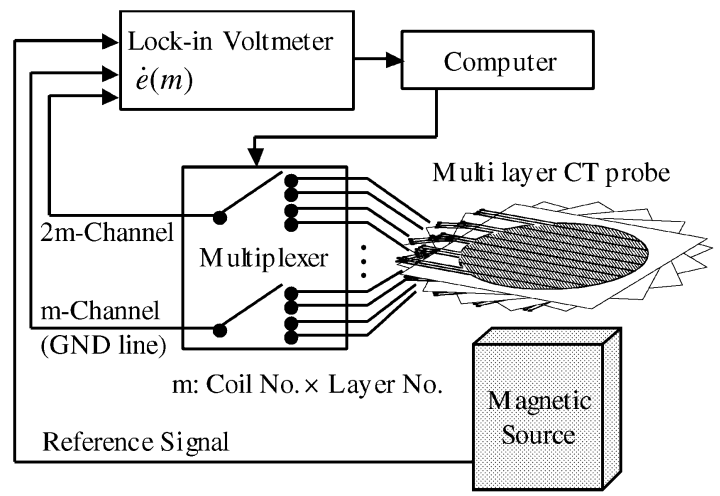

Fig. 4. Measurement system of full electronic magnetic CT probe.

probe in which many coils are arranged in parallel and the projection of the target magnetic flux under the circular plane can be evaluated.

On the other hand, (1) can be rewritten as a phasor notation under the time-varying field

$$
p \equiv \int_{u} \dot{B}(x, y) d u=-\frac{\dot{e}(v)}{j w \Delta v}
$$

where $w$ is the angular frequency of the objective field. Also $u$ and $v$ are the same variables as in (1).

From (3), the 2-D magnetic field distribution is reconstructed on a circular region. If the parameter is taken as $\Delta x=\Delta y=$ $\Delta v$, reconstructed magnetic flux distribution per unit area leads to a square of $\Delta v$. To get the reconstructed distribution by use of the FBP method, the solution is given from the following equation, where the projection data is calculated with filter function $g$

$$
\begin{aligned}
f(x, y) & =\int_{0}^{\pi}(p(u, \theta) * g(u)) d \theta \\
g(u) & =\int_{-\infty}^{\infty}|\xi| \exp (2 \pi j \xi u) d \xi
\end{aligned}
$$

where $\theta$ is the rotation angle and frequency $\xi$ is the Fourier component of $v$.

Although this equation requires probe rotation, it can reach the solution by using a multilayered CT probe, as shown in Fig. 3 [5]. In this probe, the same probes are stacked with $\Delta \theta$ pitch, and all projection data is gathered by means of switching a circuit at a time. Fig. 4 shows the schematic measurement systems. Because of the structure of the wiring between probe and multiplexer, it is easy to set this probe on the region we want to measure.

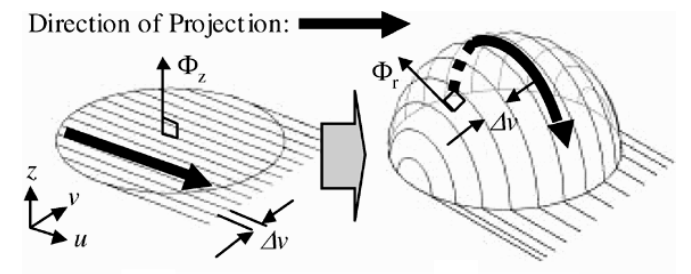

(a)

(b)

Fig. 5. From the plane-type to the spherical CT probe. (a) Plane type. (b) Spherical type.

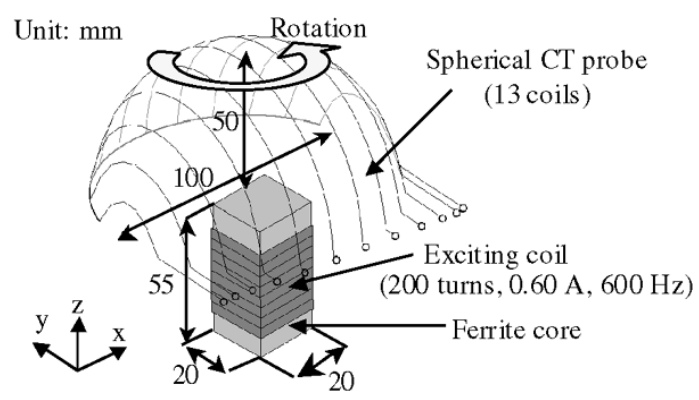

Fig. 6. Experimental target and CT probe.

\section{SPherical Magnetic CT Probe}

Basically, the line integration is always obtained along the straight line like an X-ray. But in case of the magnetic field, the line integration of magnetic flux is obtained along the coil. In other words, it is applicable to a curvilinear line by making a $\mathrm{CT}$ probe which detects radial components of the magnetic flux from the source.

Fig. 5 gives the idea of a spherical magnetic CT probe. Fig. 5(a) is the plane-type CT probe which can detect the $z$ axial direction. On the other hand, Fig. 5(b) is the spherical one which detects radial direction. By use of this spherical probe, measured data is reconstructed on the spherical surface.

Although it is difficult to measure a field on the spherical surface with other magnetic sensors by the curvilinear scanning, the multilayered spherical CT probe enables us to measure a radial magnetic field distribution with the full electronic mode and nonmechanical movement.

\section{EXPERIMENT}

In order to demonstrate the validity of spherical magnetic CT probe, a simple experiment has been carried out. Fig. 6 shows the detail of a sample object for the purpose of measurement magnetic field distribution. The probe terminals are connected with a lock-in voltmeter, and acquired ac voltage is input to the computer and calculated. Because the spherical multilayer probe has not been made yet, to obtain some angular projection data, the one-layer spherical probe is rotated mechanically in this time.

Table I shows the detail of the spherical magnetic CT probe. Fig. 7 shows the observed projection data (rms of the ac voltage), and the result of the reconstructed magnetic flux distribution on spherical surface (plotted in a Cartesian coordinate system) is shown in Fig. 8. Since the reconstruction per unit area is a little different in every place on the spherical surface, it is necessary to carry out the area correction in the postprocess. 
TABLE I

Structure of Magnetic SPHERICAl CT PRoBe

\begin{tabular}{ll}
\hline \hline Probe diameter & $\mathrm{L} \times \mathrm{W} \times \mathrm{H}=100 \mathrm{~mm} \times 100 \mathrm{~mm} \times 50 \mathrm{~mm}$ \\
Lead line & $1 \mathrm{~mm}$ width copper foil tape \\
$\begin{array}{l}\text { Coil width } \\
\text { Number of projection } \\
\text { \& back projection angle }\end{array}$ & $12 \mathrm{~mm}$ \\
Reconstruction algorithm & $\begin{array}{l}\text { Filtered back projection } \\
\text { with spline interpolation }\end{array}$ \\
\hline \hline
\end{tabular}

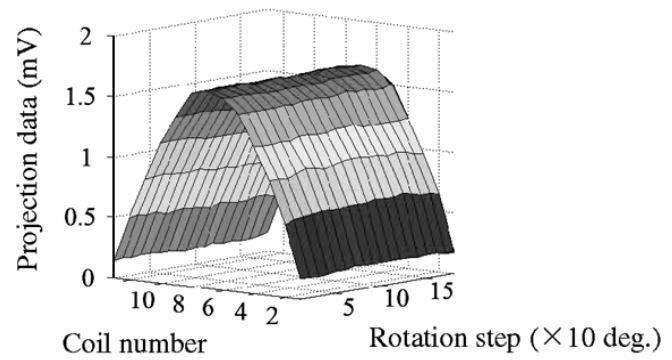

Fig. 7. Projection data of spherical magnetic CT probe.

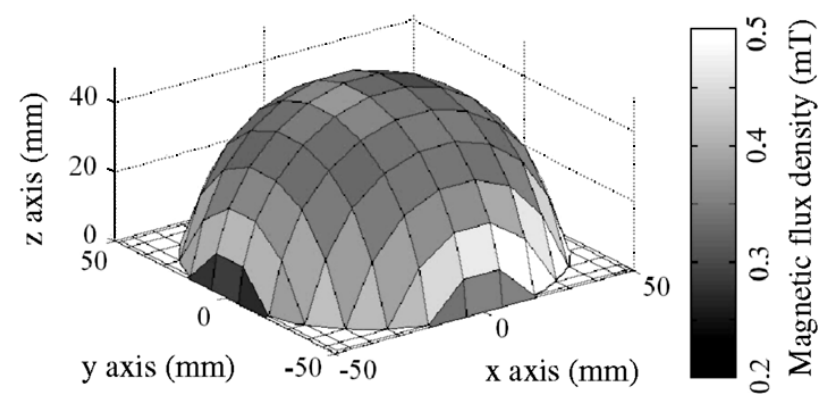

Fig. 8. Reconstructed magnetic field distribution.

To check this scheme, the reconstruction result was compared with the other two methods, where one is the search coil and the other is the finite-element method FEM (Maxwell-2D); see Fig. 6 and Table I. Fig. 9 shows the comparison result between these results on the curved line at $x=0$ in Fig. 8 , and it has been cleared that these results are in the same order.

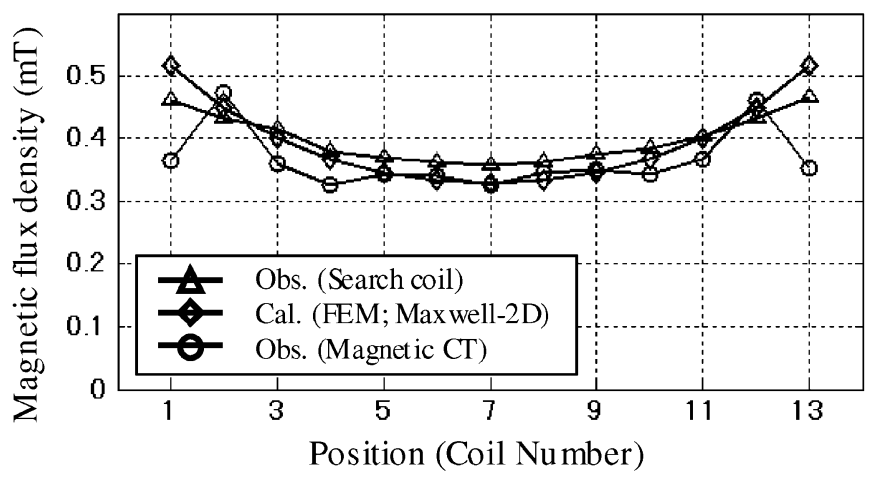

Fig. 9. Comparison between measured data and FEM result.

\section{CONCLUSION}

The measurement and visualization of a radial vectored magnetic field distribution by means of the magnetic CT method has been reported. Using this probe, a radial vectored magnetic field is measured at a time. After signal processing, the objective field distribution is reconstructed with a CT algorithm. Employing the multilayered CT probe, the procedure for getting all of the projection data can be done in the electronic mode without mechanical moving.

\section{REFERENCES}

[1] H. Saito, R. Kusaba, M. Nakajima, and S. Yuta, "Magnetic field imaging by CT technique," IEEE Trans. Magn., vol. 23, no. 5, pp. 2587-2589, Sep. 1987.

[2] A. C. Kak and M. Slaney, Principles of Computerized Tomographic Imaging. New York: IEEE Press, 1988.

[3] M. Iwahara and S. Yamada, "Visualization of magnetic field by means of the projection method with signed objectives," J. Magn. Magn. Mater. vol. 272-276, pp. 2263-2265, 2004.

[4] D. Ebihara, J. Tsutiya, S. Wakui, and S. Inui, "The activity reports of investigation committee on the multi-direction drive systems," Rec. IEE-J. Technical Meeting Linear Drives, 2003. LD-03-34.

[5] K. Tashiro, N. Nakamura, M. Szumilo, M. Iwahara, and S. Yamada, "Visualization of magnetic flux distribution using multi layered search coil and CT method," Proc. Non-Linear Electromagnetic System, ISEM'99, pp. 253-256, 2000.

Manuscript received February 7, 2005. 